\title{
Comparison of three methods for the methylation of aliphatic and aromatic compounds
}

Hyejung Lee ${ }^{1 *}$, Sarah J. Feakins ${ }^{1}$, Zhiyao Lu², Arndt Schimmelmann ${ }^{3}$, Alex L. Sessions ${ }^{4}$, Jessica E. Tierney ${ }^{5}$, Travis J. Williams ${ }^{2}$

${ }^{1}$ Department of Earth Sciences, University of Southern California, Los Angeles, CA 90089, USA

${ }^{2}$ Department of Chemistry, University of Southern California, Los Angeles, CA 90089, USA

${ }^{3}$ Department of Earth and Atmospheric Sciences, Indiana University, Bloomington, IN 47405-1405, USA

${ }^{4}$ Division of Geological and Planetary Sciences, California Institute of Technology, Pasadena, CA 91125, USA

${ }^{5}$ Department of Geosciences, University of Arizona, Tucson, AZ 85721, USA

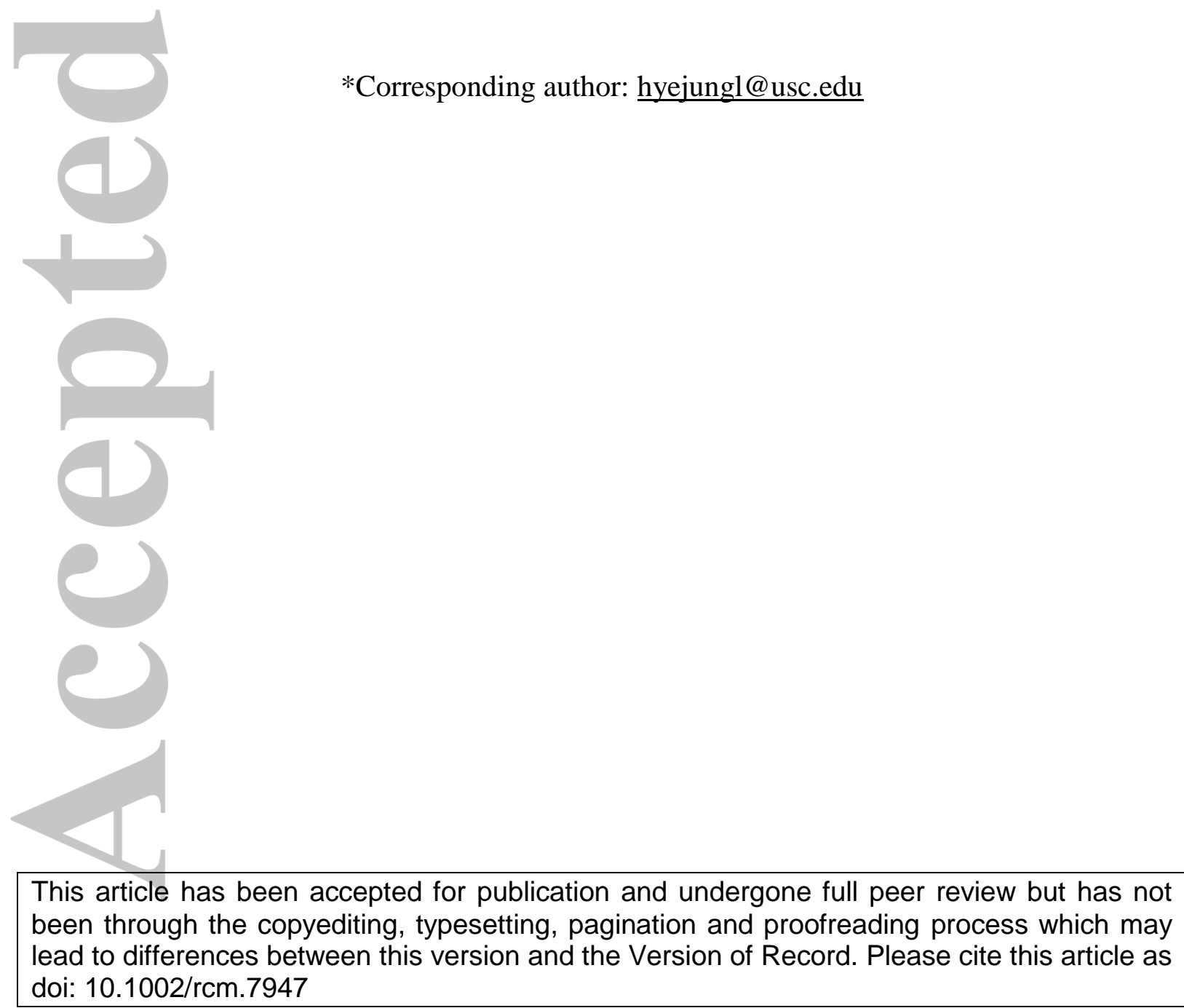

This article is protected by copyright. All rights reserved. 


\section{Abstract}

\section{Rationale}

Methylation protocols commonly call for acidic, hot conditions that are known to promote organic ${ }^{1} \mathrm{H} /{ }^{2} \mathrm{H}$ exchange in aromatic and aliphatic $\mathrm{C}-\mathrm{H}$ bonds. Here we tested two such commonly-used methods and compared a third that avoids these acidic conditions, to quantify isotope effects with each method and to directly determine acidic-exchange rates relevant to experimental conditions.

\section{Methods}

We compared acidic and non-acidic methylation approaches catalyzed by hydrochloric acid, acetyl chloride and EDCI (1-ethyl-3-(3-dimethylaminopropyl)carbodiimide) / DMAP (4dimethylaminopyridine) respectively. These were applied to two analytes: phthalic acid (an aromatic) and octacosanoic acid (an aliphatic). We analyzed yield by gas chromatography flame ionization (GC/FID) and hydrogen and carbon isotopic composition by isotope ratio mass spectrometry (GC/IRMS). We quantified the ${ }^{1} \mathrm{H} /{ }^{2} \mathrm{H}$ exchange rate on dimethyl phthalate under acidic conditions with proton nuclear magnetic resonance $\left({ }^{1} \mathrm{H}-\mathrm{NMR}\right)$ measurements.

\section{Results}

The $\delta^{2} \mathrm{H}$ and $\delta^{13} \mathrm{C}$ values and yield were equivalent among the three methods for methyl octacosanoate. The two acidic methods resulted in comparable yield and isotopic composition of dimethyl phthalate; however, the non-acidic method resulted in lower $\delta^{2} \mathrm{H}$ and $\delta^{13} \mathrm{C}$ values perhaps due to low yields. Concerns over acid-catalyzed ${ }^{1} \mathrm{H} /{ }^{2} \mathrm{H}$ exchange are unwarranted as the effect was trivial over a 12-hour reaction time.

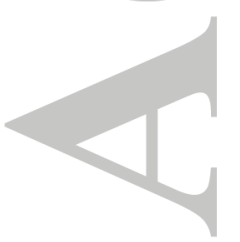




\section{Conclusions}

We find product isolation yield and evaporation to be the main concerns in the accurate determination of isotopic composition. ${ }^{1} \mathrm{H} /{ }^{2} \mathrm{H}$ exchange reactions are too slow to cause measurable isotope fractionation over the typical duration and reaction conditions used in methylation. Thus, we are able to recommend continued use of acidic catalysts in such methylation reactions for both aliphatic and aromatic compounds.

\section{INTRODUCTION}

Stable hydrogen isotopic compositions ( $\delta^{2} \mathrm{H}$ values) of organic compounds are widely used in organic geochemistry, biochemistry, ecology, climate, and forensics. Applications include the

study of the hydrologic cycle and climate,${ }^{[1-3]}$ ecology,${ }^{[4-6]}$ biosynthetic pathways of lipids, ${ }^{[7,8]}$ paleoelevation, ${ }^{[9,10]}$ diagenesis of organic matter, ${ }^{[11]}$ and food authenticity and provenance. ${ }^{[12,13]}$ In many compounds, hydrogen positions include those in $\mathrm{C}-\mathrm{H}$ bonds that are non-exchangeable after biosynthesis and preserved under low temperature sedimentary conditions. They carry the original biosynthetically fixed $\mathrm{H}$, although even these $\mathrm{H}$ may be exchanged under elevated temperatures and over geologic time. ${ }^{[14,15]}$ In contrast, $\mathrm{O}-\mathrm{H}$ bonds, as found in cellulose or fatty acids, contain $\mathrm{H}$ atoms that are readily exchangeable with ambient water in the plant, ${ }^{[16]}$ soil, or sediments after burial. ${ }^{[11]}$ In order to recover signals relating to the original plant biosynthesis, analyses typically target only the carbon-bound hydrogens, by masking exchangeable $\mathrm{O}-\mathrm{H}$ hydrogens. The preparative steps for cellulose involve 'nitration' (i.e. formation of nitric acid esters ${ }^{[17]}$ ), whereas derivatization of aliphatic and aromatic acids typically involves esterification with methanol. Here, we examine isotopic exchange associated with reaction conditions used for esterification prior to isotopic analysis. 


\section{Acid catalyzed esterification}

Organic compounds with carboxylic acid groups are commonly esterified with methanol prior to gas chromatography (GC) analysis ${ }^{[18,19]}$ using acidic catalysts. Hydrochloric acid $(\mathrm{HCl})$ and acetyl chloride $\left(\mathrm{CH}_{3} \mathrm{COCl}\right)$, which forms anhydrous $\mathrm{HCl}$ in methanol, are among the most widely used catalysts of aliphatic and aromatic acids. The carboxylic acid is initially protonated and undergoes an exchange reaction with the alcohol to give the reaction intermediate. The intermediate can then lose a proton to become an ester. The equilibrium point of this reaction is displaced so that esterification proceeds virtually to completion in excess alcohol. ${ }^{[20]}$ However, Brønsted acid catalyst can also promote hydrogens bound to an aromatic ring to undergo ${ }^{1} \mathrm{H} /{ }^{2} \mathrm{H}$ exchange via electrophilic aromatic substitution (Figure 1). ${ }^{[21-23]}$ Thus, during esterification, hydroxyl hydrogens in methanol can exchange with aromatic hydrogens in an analyte molecule and the $\delta^{2} \mathrm{H}$ values can equilibrate.

Understanding acid-catalyzed isotope exchange during esterification is relevant to the study of aromatic compounds, but also indirectly for the study of aliphatic compounds such as the alkanoic acids derived from plant leaf waxes and microbial lipids, because aromatic structures are commonly used as part of the isotopic determination. ${ }^{[24]}$ One method to determine the $\delta^{2} \mathrm{H}$ values and the carbon isotopic composition $\left(\delta^{13} \mathrm{C}\right.$ values $)$ of methyl groups added in alkanoic acid esterification steps involves the use of phthalic acid. ${ }^{[25]}$ Phthalic acid of known isotopic composition (measured independently as a sodium salt) can be methylated and measured by gas chromatography isotope ratio mass spectrometry (GC/IRMS) to calculate the isotopic composition of the methyl hydrogens and carbon via mass balance. ${ }^{[19]}$ Because phthalic acid has an aromatic ring, conventional acidic esterification conditions should enable ${ }^{1} \mathrm{H} /{ }^{2} \mathrm{H}$ exchange on the aromatic hydrogens (Figure 1). ${ }^{[21-23]}$ Such exchange may result in an incorrect estimation of the overall dimethyl phthalate isotopic composition 
and mass balance estimation of the $\delta^{2} \mathrm{H}$ value of methyl hydrogens, needed when calculating the isotopic composition of the original fatty acid. ${ }^{[26]}$ However, the magnitude of such an effect is currently unknown.

\section{Non-acidic coupling reagents to promote esterification}

Alternatively, non-acidic approaches to methylation exist that may obviate the above concerns. In this study, we tested a non-acidic method using 1-ethyl-3-(3dimethylaminopropyl)carbodiimide (EDCI) as a coupling reagent with 4dimethylaminopyridine (DMAP) as the catalyst (Figure 2). ${ }^{[27]}$ Carbodiimides, due to their accessibility and versatility, are ranked as one of the most important classes of compounds in organic chemistry. ${ }^{[28]}$ The most widely used carbodiimide reagent, dicyclohexylcarbodiimide (DCC), makes insoluble urea byproducts requiring additional cumbersome purifications. ${ }^{\text {[29] }}$ Hence we chose EDCI, a water soluble carbodiimide with water soluble urea byproduct, ${ }^{[30]}$ combined with DMAP which has been shown to increase the efficiency of esterification by $10^{4}$ fold. ${ }^{[31]}$ We compared this approach with conventional acidic methylation reactions. EDCI and DMAP are not expected to lead to ${ }^{1} \mathrm{H} /{ }^{2} \mathrm{H}$ exchange because the reaction conditions are buffered, and the merits of these reagents for organic geochemical applications are tested here.

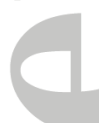

\section{Method comparison}

In this study, we tested two acidic and one non-acidic methods of esterification as applied to phthalic acid and octacosanoic acid to evaluate any isotopic fractionations and practical issues arising with each using GC/IRMS and GC/FID approaches. We further directly measured the ${ }^{1} \mathrm{H} /{ }^{2} \mathrm{H}$ exchange rate in phthalic acid methylation with 5 vol\% $\mathrm{HCl}$ using proton nuclear magnetic resonance $\left({ }^{1} \mathrm{H}-\mathrm{NMR}\right)$. Based on the exchange rate, we estimate the 
proportion of aromatic $\mathrm{H}$ substituted over 12 hours of reaction time from the initial $\delta^{2} \mathrm{H}$ values of the reactants. The goal is to quantify the $\delta^{2} \mathrm{H}$ and $\delta^{13} \mathrm{C}$ perturbation possible with each esterification method and to make practical recommendations for esterification prior to GC/IRMS analyses.

\section{EXPERIMENTAL}

\section{Phthalic acid and methanol of known isotopic composition}

The hydrogen isotopic composition of phthalate was measured in Na-phthalate to exclude exchangeable, carboxylic acid hydrogens and the $\delta^{13} \mathrm{C}$ value was measured from the free diacid via offline combustion at Indiana University (Bloomington, IN, USA). Offline combustion consisted of phthalic acid combustion in quartz ampoules and cryogenic purification of combustion gases in a vacuum line. The aromatic hydrogen isotopic composition was $-95.5 \pm 2.2 \%(\sigma, \mathrm{n}=4)$ and the carbon had a $\delta^{13} \mathrm{C}$ value of $-27.21 \pm 0.02$ $\%$ o $(n=4)$. The uncertainties reported represent replicate precision. Unquantified uncertainty in the absolute value may exist, and the largest source of concern would be water inclusion in the Na-phthalate crystals. In order to mitigate against the influence of water inclusions, the crystals are warmed before combustion.

Methanol $(\mathrm{MeOH})$ was $99.8 \%$ anhydrous and the $\delta^{2} \mathrm{H}$ value of the methyl group was $-141 \pm$ $3 \%(n=3)$, calculated via mass balance between bulk methanol and hydroxyl hydrogen at Indiana University. Excess sodium metal and a small amount of methanol were reacted in an evacuated, sealed ampoule to liberate $\mathrm{H}_{2}$ gas from the hydroxyl hydrogen for isotopic determination. The uncertainties reported represent replicate precision. Unquantified uncertainty in the absolute value may exist, the largest source of such error would be associated with residual methanol vapor, although this is mitigated against by allowing the 
reaction to proceed for several days. The hydroxyl hydrogen has a $\delta^{2} \mathrm{H}$ value of $-27 \%$ and thus can provide the relatively ${ }^{2} \mathrm{H}$-enriched hydrogen source for exchange into the phthalate aromatic ring during acid-catalyzed equilibration. The other $\mathrm{H}^{+}$in the 5 vol\% catalyst (from $\mathrm{HCl}$ and water) can exchange freely with methanol's hydroxyl hydrogen and their isotopic compositions are unknown. Nonetheless, the dominant source of exchangeable hydrogens is from methanol, which is $95 \%$ of the reaction solvent. The $\delta^{13} \mathrm{C}$ value of the methanol was $-46.77 \pm 0.04 \%$, from bulk measurement. The isotopic compositions of phthalic acid and methanol were used to calculate the predicted isotope values of dimethyl phthalate via Eq. 1 for hydrogen and Eq. 2 for carbon.

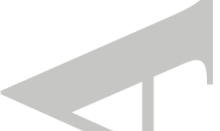

$\delta^{2} H$ dimethyl phthalate $H \times 10=\delta^{2} H$ phthalic acid $H \times 4+\delta^{2} H$ methyl $H \times 6$

$\delta^{13} C$ dimethyl phthalate $C \times 10=\delta^{13} C$ phthalic acid $C \times 8+\delta^{13} C$ methyl $C \times 2$

\section{The acidic methods: $\mathrm{HCl}$ and $\mathrm{CH}_{3} \mathrm{COCl}$}

For the $\mathrm{HCl}$ catalyzed methylation, $3 \mathrm{mg}$ of phthalic acid or $3 \mathrm{mg}$ of $\mathrm{C}_{28} n$-alkanoic acid were methylated with $1 \mathrm{~mL}$ of a mixture of $5 \mathrm{vol} \% \mathrm{HCl}$ and $95 \% \mathrm{MeOH}$ in a tightly sealed $15-\mathrm{mL}$ culture tube at $70{ }^{\circ} \mathrm{C}$ overnight. For the acetyl chloride catalyzed methylation, the same procedure was followed using $\mathrm{CH}_{3} \mathrm{COCl}$ (instead of $\mathrm{HCl}$ ) and reacting at $50{ }^{\circ} \mathrm{C}$ overnight. Practical guidance on methylation protocols are provided in the supplementary material, and are briefly summarized here.

Following overnight methylation reactions, the samples were removed from the heat, and 1 mL of Milli-Q water (Millipore Milli-Q Plus QPAK 2; Millipore, Billerica, MA, USA) was then added to produce an aqueous phase. The analyte was extracted using liquid-liquid extraction with $1 \mathrm{~mL}$ of dichloromethane (DCM) for dimethyl phthalate and hexane for $n$ - 
alkanoic acid methyl esters. For each extraction (repeated 3 or more times), the mixture was vigorously shaken, left to partition into two phases and the organic phase, containing dimethyl phthalate or fatty acid methyl ester (FAME), was extracted by pipette and dried by passage through a column of anhydrous $\mathrm{Na}_{2} \mathrm{SO}_{4}$. The organic fraction was further purified by passing through a silica column $(5 \mathrm{~cm} \times 40 \mathrm{~mm}$ Pasteur pipette, $5 \mathrm{wt} \%$ water-deactivated silica gel, 100-200 mesh) eluting with three column volumes of DCM for dimethyl phthalate and for FAME.

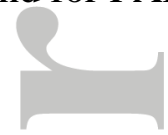

\section{The non-acidic method: EDCI and DMAP}

A general procedure for synthesis of ester derivatives in Lehmann et al ${ }^{[27]}$ was followed, with adjustments for scale. $22.5 \mathrm{mg}$ of EDCI ( 8 mol ratio to phthalic acid or octacosanoic acid) and $1.1 \mathrm{mg}$ of DMAP $\left(0.5 \mathrm{~mol}\right.$ ratio) and $3 \mathrm{mg}$ of phthalic acid or octacosanoic acid $\left(\mathrm{C}_{28} n\right.$ alkanoic acid) were added to $1 \mathrm{~mL}$ of isotopically known methanol and the solution was kept at room temperature overnight. The solution was diluted with $1 \mathrm{~mL}$ of Milli-Q water. The methylated product was extracted through the addition of $1 \mathrm{~mL}$ of ethyl acetate. The mixture was shaken vigorously to allow liquid:liquid extraction to proceed, and then allowed to settle into two phases, and this process was repeated at least three times. The organic fraction, containing dimethyl phthalate or methyl octacosanoate, was dried by passage through a column of anhydrous $\mathrm{Na}_{2} \mathrm{SO}_{4}$. It was further purified by eluting the ester fraction through a silica column with three column volumes of ethyl acetate.

\section{GC/MS/FID analysis}

The methylation products generated in this study, including dimethyl phthalate and $\mathrm{C}_{28}$ FAME, were identified and quantified using gas chromatography (Agilent 6890 series) coupled with a single quadrupole mass-selective detector (Agilent 5973), using electron 
ionization with an ionization energy of $69.9 \mathrm{eV}$ ) and flame ionization detection (GC/MS/FID instrumentation supplied by Agilent, Santa Clara, CA, USA). The instrument was equipped with a Rxi-5 ms column ( $30 \mathrm{~m} \times 0.25 \mathrm{~mm}$, film thickness $0.25 \mu \mathrm{m}$ supplied by Restek Corporation, Bellefonte, PA, USA) with a $4 \mathrm{~mL} \mathrm{~min}^{-1}$ constant column flow rate, split passively 4:1 (based on theoretical calculations following Poiseulle's law) between the mass spectrometer (via a $80 \mathrm{~cm} \times 0.25 \mathrm{~mm}$ silica capillary) and the flame ionization detector (via

a $120 \mathrm{~cm} \times 0.1 \mathrm{~mm}$ silica capillary). Helium was used as the carrier gas. The initial

temperature of $50{ }^{\circ} \mathrm{C}$ was held for $3.5 \mathrm{~min}$, followed by a temperature ramp of $20{ }^{\circ} \mathrm{C} \mathrm{min}-1$ to $300^{\circ} \mathrm{C}$ and maintained for $10 \mathrm{~min} .1 \mu \mathrm{L}$ of sample was injected in splitless mode. Absolute abundance was calculated using a calibration curve of an in-house standard (mixture of $n$ alkanes and $n$-alkanoic acids) and their peak area response on the flame ionization detector. Yields were calculated based on the chemical equation using masses of reagents, quantified product abundances and are reported as $\%$ of predicted product. All uncertainties are reported as standard deviations $(\sigma)$ unless otherwise stated.

\section{GC/IRMS analysis}

Compound-specific hydrogen and carbon isotopic compositions were measured using GC/IRMS. Samples were introduced via a programmable temperature vaporizing (PTV) injector in splitless mode at $50{ }^{\circ} \mathrm{C}$ to a Thermo Scientific ${ }^{\circledR}$ Trace gas chromatograph. The gas chromatograph was equipped with a Rxi-5 ms column $(30 \mathrm{~m} \times 0.25 \mathrm{~mm}$, film thickness 0.25 $\mu \mathrm{m})$ and connected via an Isolink with a pyrolysis furnace at $1400{ }^{\circ} \mathrm{C}$ for hydrogen isotopic analysis, and via a combustion furnace at $1000{ }^{\circ} \mathrm{C}$ for carbon isotopic analysis, through a Conflo IV interface to a DeltaV ${ }^{\text {Plus }}$ isotope ratio mass spectrometer (all supplied by Thermo 
Scientific, Bremen, Germany). The gas chromatograph was operated with a He flow rate of 1 $\mathrm{mL} \min ^{-1}$ from an initial temperature of $50{ }^{\circ} \mathrm{C}$, followed by a ramp of $20{ }^{\circ} \mathrm{C} \min ^{-1}$ to $300{ }^{\circ} \mathrm{C}$, and held for $5 \mathrm{~min}$.

For hydrogen, the $\mathrm{H}_{3}{ }^{+}$factor was measured daily to correct for the formation of $\mathrm{H}_{3}{ }^{+}$in the ion source $^{[32]}$, and to check for linearity, with a mean value over the course of measurements reported here of $3.6 \mathrm{ppm} \mathrm{mV}^{-1} \pm 0.35(\mathrm{n}=6)$ across a range of $1-8 \mathrm{~V}$. For carbon, the standard deviation of $\mathrm{CO}_{2}$ pulses spanning 1-8 $\mathrm{V}$ was determined daily to check for linearity and the standard deviation across that range averaged $0.02 \%$. Gas peaks of $\mathrm{H}_{2}$ or $\mathrm{CO}_{2}$ as appropriate were co-injected bracketing the analyte peaks during the GC/IRMS run. Two of these peaks were used for standardization between sample and standard, while the rest were treated as unknowns to assess precision. Data were then normalized to the Vienna Standard Mean Ocean Water (VSMOW)/Standard Light Antarctic Precipitation (SLAP) hydrogen isotopic scale via comparison with an external standard with $15 n$-alkanes $\left(\mathrm{C}_{16}-\mathrm{C}_{30} n\right.$-alkane mixture of type $\mathrm{A}$, isotopic standard from Indiana University), with $\delta^{2} \mathrm{H}$ values ranging from -46 to $-227 \%$. For carbon, the isotopic results were normalized to Vienna PeeDee Belemnite (VPDB) / lithium carbonate prepared by H. Svec (LSVEC) by comparing with A mix standard $\delta{ }^{13} \mathrm{C}$ values ranging from -28.6 to $-33.3 \%$. The standard was run daily throughout the sequence. Measured $\delta^{2} \mathrm{H}$ values of the samples are normalized using a regression between the known and measured values of the standards. The root-mean-square error of replicate analysis of the external standard (A mix) averaged $4.6 \%$ for hydrogen and $0.2 \%$ for carbon. The results are reported using conventional delta $(\delta)$ notation in permil (\%o).

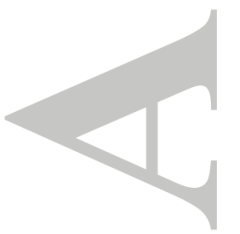




\section{${ }^{1} \mathrm{H} /{ }^{2} \mathrm{H}$ exchange rate using ${ }^{1} \mathrm{H}-\mathrm{NMR}$}

We conducted a proton nuclear magnetic resonance $\left({ }^{1} \mathrm{H}-\mathrm{NMR}\right)$ kinetic experiment to measure the rate of ${ }^{1} \mathrm{H} /{ }^{2} \mathrm{H}$ exchange on dimethyl phthalate in 5 vol\% $\mathrm{HCl}(12 \mathrm{M})$ using a Varian VNMRS-600 (supplied by Varian, Inc. now Agilent Technologies, Palo Alto, CA, USA) at the University of Southern California (Loa Angeles, CA, USA). We reacted $30 \mathrm{mg}$ of phthalic acid in a solution of $0.60 \mathrm{~mL}$ of methanol- $d_{4}\left(\mathrm{C}^{2} \mathrm{H}_{3} \mathrm{O}^{2} \mathrm{H}\right)$ and $5 \mathrm{vol} \% \mathrm{HCl}$ in a Wilmad J Young NMR tube (type of NMR tube with a Teflon cap that can be attached to a vacuum line, supplied by Wilmad-labglass, Vineland, NJ, USA) sealed with plastic cap and parafilm, in an oil bath at $70{ }^{\circ} \mathrm{C}$. We measured the phthalate $\mathrm{H}$ peak areas daily for ten days after the overnight esterification reaction. The tube was placed back in the oil bath after measurement to maintain the reaction conditions over the ten days. The peaks were analyzed with a relaxation delay of $10 \mathrm{sec}$. A decrease in peak area detected by ${ }^{1} \mathrm{H}-\mathrm{NMR}$ indicated exchange of ${ }^{1} \mathrm{H}$ on the phthalate with ${ }^{2} \mathrm{H}$ from the methanol- $d_{4}$ solvent.

\section{RESULTS AND DISCUSSION}

\section{Product yield}

We found that product yields varied between methods and analytes. For dimethyl phthalate, the acidic methods resulted in $>70 \%$ product yield, whereas the carbodiimide method resulted in yields $<30 \%$ (Fig. 3, Supplementary Table 1, supplementary material). For methyl octacosanoate, all methods yielded $>80 \%$ product yield, but again yields were highest for the acidic methods $>94 \%$ (Supplementary Table 2, supplementary material). Low product yield (observed here for the dimethyl phthalate using the non-acidic method) is a concern given the potential for isotopic fractionation associated with incomplete reaction or recovery. Low yields are also problematic for many applications because sample size 
requirements increase. We investigate here the potential reasons for low yields and discuss our attempts to improve them.

\section{Yield for acidic methods}

High yields were obtained using acidic methods for methylation, with acetyl chloride (mean yield $80 \%, \sigma=5, \mathrm{n}=10$ ) or $\mathrm{HCl}$ (mean yield $73 \%, \sigma=5, \mathrm{n}=13$ ) as the starting reagent, consistent with theoretical product yield for Fischer esterification. ${ }^{[20]}$ The higher yield for the acetyl chloride method is due to the lower water content of the reagent, compared with $\mathrm{HCl}$ (12 M solution). These high yields were achieved after experimentation to optimize handling to minimize loss (Supplementary Table 3, supplementary material). For example, the boiling point of dimethyl phthalate is $283{ }^{\circ} \mathrm{C}$ and we observed significant removal of the product by GC/FID under normal solvent blow-down on a hotplate; thus, we avoided using the hotplate. We also improved our yield by up to $10 \%$ by switching from hexane (used for aliphatic esters) to DCM during liquid-liquid extraction, given the greater polarity of dimethyl phthalate. Still, we found dimethyl phthalate has a lower isolated yield than aliphatic esters (Supplementary Table 1, supplementary material), suggesting that extraction and purification of dimethyl phthalate could be further improved. Less than $100 \%$ isolated yield could be due to either incomplete liquid-liquid extraction or evaporative loss prior to measurement.

\section{Yield for carbodiimide method}

The isolated yield of dimethyl phthalate was low (mean $22 \%$ ) using the carbodiimide method and this could be due to incomplete reaction or loss during purification. We monitored the reaction with thin layer chromatography (TLC) at the beginning and end of the reaction (after $12 \mathrm{hrs}$ ) and we confirmed that the product had formed while phthalic acid had been completely consumed. Additional evidence for reaction completion was obtained by testing 
for the absence of phthalic anhydride, a reaction intermediate for the carbodiimide method, at the end of the experiment by GC/MS. Thus, we can rule out incomplete reaction.

Low product yield using carbodiimide reagents for esterification has been previously noted, due to the carboxylate being consumed during the formation of an $N$-acylurea byproduct ${ }^{[33]}$. Addition of DMAP as a catalyst inhibits the byproduct formation and facilitates the reaction to form the ester, which has been shown to increase yield. ${ }^{[31]}$ Following this protocol, we obtained $<30 \%$ expected yield of dimethyl phthalate, while obtaining $>80 \%$ yield of methyl octacosanoate. The low yield of dimethyl phthalate may be explained by its greater volatility than methyl octacosanoate.

We tested yield response to various reaction conditions such as reagent concentration, temperature and duration of reaction for the carbodiimide approach, although tests of intermediates showed that reactions went to completion. In case isolation and purification steps were the issues affecting low isolation yields we tested different solvents for extraction, as well as citric acid washing and brine washing in efforts to improve the isolated product yield. Each of these efforts failed to generate yield $>30 \%$ (Supplementary Table 4, supplementary material). Having experimented considerably with this approach, we find it is not currently a pragmatic protocol for low concentration ( $3 \mathrm{mg}$ ) dimethyl phthalate derivatization applications. In contrast, the method does have promise as a viable method for derivatization of aliphatic acids.

Nevertheless, we note additional practical challenges for the use of the carbodiimide method prior to GC analyses. We found it necessary to clean the syringe, inlet and trim the column after running the carbodiimide samples by GC/IRMS probably due to urea persisting in the 
mixture even after liquid-liquid extraction. If these compounds were injected in measurable quantity, due to their high polarizability and protic nature, they could interact with the GC

column. We do not, however, observe any humps or fronting and tailing of the analyte peak in the chromatogram; our analytical results for both quantification and isotopic composition therefore appear to be unaffected by any such polar contaminants. Regardless of analyte, the carbodiimide method presented challenges for subsequent GC analyses that should be addressed if this approach is to be adopted for aliphatic GC applications.

\section{Isotopic Analyses}

The expected isotopic ratios of dimethyl phthalate calculated from mass balance (Eq. 1 and Eq. 2) were $\delta^{2} \mathrm{H}$ values of $-123 \%$ and $\delta^{13} \mathrm{C}$ values of $-31.1 \%$. For carbon isotopes, acidic methods successfully resulted in values that are close to those expected (Figure 3). Acetyl chloride and $\mathrm{HCl}$ methods produced $\delta^{13} \mathrm{C}$ values of $-31.5 \pm 0.0 \%(\mathrm{n}=3)$ and $-31.3 \pm 0.1 \%$ o $(n=3)$. The non-acidic method resulted in a more negative value of $-32.7 \pm 0.4 \%(n=4)$.

The hydrogen isotopic composition of dimethyl phthalate differed between the acidic and non-acidic esterification methods (Figure 3). Dimethyl phthalate produced with acetyl chloride and $\mathrm{HCl}$ resulted in $\delta^{2} \mathrm{H}$ values of $-105 \pm 2 \%$ (n $\left.=3\right)$ and $-107 \pm 1 \%$ o $(\mathrm{n}=3)$, respectively, thus being equal within uncertainty although +16 to $+18 \%$ more ${ }^{2} \mathrm{H}$-enriched than expected. The carbodiimide method resulted in a more negative $\delta^{2} \mathrm{H}$ value of $-137 \pm 1$ $\% 0(n=4)$. We calculated the initial methyl hydrogen and carbon isotopic composition of methanol ${ }^{[25]}$ by mass balance and found that none of the methods accurately assessed the (known) initial methyl $\delta^{2} \mathrm{H}$ and $\delta^{13} \mathrm{C}$ values. Acidic methods produced $25-29 \%$ more positive estimates for methyl $\delta^{2} \mathrm{H}$ values whereas the carbodiimide method produced $25 \%$ more negative estimates than the known value of the methanol $-141 \pm 3 \%, n=3$ (Table 1 ). 
This inaccuracy is greater than analytical errors, the largest of which is the calibration to the international VSMOW-SLAP isotopic scale (see Methods). This suggests an isotopic

fractionation during the methylation reaction and/or purification and one that differs between the acidic and non-acidic methods.

\section{Is acid-catalyzed exchange a cause of ${ }^{2} H$-enrichment?}

We directly measured ${ }^{1} \mathrm{H} /{ }^{2} \mathrm{H}$ exchange rates for dimethyl phthalate by ${ }^{1} \mathrm{H}-\mathrm{NMR}$ under conditions relevant to the acidic methylation method. We found the same exchange rates (slopes) within uncertainties for the hydrogens on the alpha and beta carbon positions of dimethyl phthalate (Figure 4). Using the mean slope as the exchange rate constant $(4.94 \times$ $10^{-4} \mathrm{hr}^{-1}$ ), this corresponds to an exchange half-life (the time to exchange $50 \%$ of aromatic hydrogens, $\left.=\frac{\ln (2)}{k}\right)$ of 1403 hours $(58.5$ days $)$ at $70{ }^{\circ} \mathrm{C}$. For a $100 \%$ disequilibrium in $\delta^{2} \mathrm{H}$ values between aromatic $\mathrm{H}$ and methanol hydroxyl $\mathrm{H}$, this would induce a change in the $\delta^{2} \mathrm{H}$ value of the aromatic $\mathrm{H}$ of just $0.035 \%$ over a 1 -hour reaction, which is not detectable by GC/IRMS. For the reagents described here, with $70 \%$ disequilibrium in the $\delta^{2} \mathrm{H}$ values of dimethyl phthalate and methanol hydroxyl $\mathrm{H}$, and a 12 -hour reaction time ( $0.4 \%$ exchange), we would expect $0.3 \%{ }^{2} \mathrm{H}$-enrichment. Even with a 12 -hour reaction ( $0.4 \%$ exchange) and a $1000 \%$ disequilibrium, such as might be encountered working on extraterrestrial samples, a shift of only $4 \%$ is predicted, which is still smaller than the analytical uncertainties for GC/IRMS. Although ${ }^{1} \mathrm{H} /{ }^{2} \mathrm{H}$ exchange is taking place as expected, we directly establish, for the first time, that the rates are too slow to cause any significant changes in $\delta$-values over the reaction conditions used in the $\mathrm{HCl}$ method. Still, when using strong acids as catalysts in methylation, it is good practice to not extend reaction times. 
Is evaporation a cause of ${ }^{2}$ H-fractionation?

Guarding against evaporation is always a concern for volatile analytes intended for isotopic analyses, requiring careful laboratory handling. Vapor pressure isotope effects vary between molecules, depending on intermolecular vibrations (bonding) and frequency shifts on condensation, both of which vary with temperature. ${ }^{[34]}$ We directly tested fractionation for dimethyl phthalate under worst-case scenario laboratory handling with evaporative losses to demonstrate the scale of possible errors. To mimic extreme sample preparation error, we evaporatively removed all solvent and $\sim 45 \%$ of the dimethyl phthalate using $\mathrm{N}_{2}$ blowdown, redissolved the compound in solvent and observed $+14 \%{ }^{2} \mathrm{H}$-enrichment upon reanalysis. We also tested GC inlet conditions: we compared $\delta^{2} \mathrm{H}$ values of dimethyl phthalate injected via a PTV inlet in splitless mode with those from split-mode at $150^{\circ} \mathrm{C}$. With the inletevaporation test, the peak area decreased by half and we measured a ${ }^{2} \mathrm{H}$-enrichment of $+8 \%$. These tests indicate that it is possible to fractionate dimethyl phthalate during handling, such as complete solvent blow-down, or during analysis, via the use of heated, split injection modes. To avoid such effects, we dried the solvents under a gentle $\mathrm{N}_{2(\mathrm{~g})}$ stream without the use of heat and ran samples in splitless injection mode. Yields were still below $100 \%$ for acidic methods, leaving open the possibility that some product evaporated.

\section{${ }^{2} \mathrm{H}$-and ${ }^{13} \mathrm{C}$-depletion using the carbodiimide method}

The carbodiimide method resulted in more negative $\delta^{2} \mathrm{H}$ and $\delta^{13} \mathrm{C}$ values than expected for dimethyl phthalate compared with the known isotopic composition. We infer a kinetic isotope effect, with the preferential reaction of the lighter isotope, by the carbodiimide method. ${ }^{[35]}$ Although we have not fully resolved the cause of the isotopic offset using this method (Figures 3 and 4) we suggest that securing higher yields and determining the potential for 
kinetic isotope effects with the carbodiimide reaction may be relevant questions for future research if this method is to be used as preparative method prior to GC/IRMS analyses.

\section{Implications for aromatic and aliphatic acid esterification}

For methyl octacosanoate, prepared by each of three methods, the $\delta^{2} \mathrm{H}$ and $\delta^{13} \mathrm{C}$ values were within $3 \%$ and $0.5 \%$ uncertainty envelopes, respectively (Figure 5). Thus, all three methods are suitable preparative methods for GC/FID and GC/IRMS applications for long chain fatty acids, although the acidic methods remain the recommended ones.

For phthalic acid, we found that the two acidic methods are equivalent, and $\delta^{13} \mathrm{C}$ values were as predicted (within $0.4 \%$, similar within uncertainties), although we found that the $\delta^{2} \mathrm{H}$ values were elevated by $15 \%$ (Figure 3 ). Although acidic exchange can lead to ${ }^{2} \mathrm{H}$ enrichment, the rates established here are far too slow to explain observations. Small ${ }^{2} \mathrm{H}-$ enrichment can be reproduced through evaporation; however, evaporation cannot fully explain the observed enrichment given the high yields obtained, given that $\sim 50 \%$ of the analyte would have to be lost to explain such an enrichment. Thus, the remaining offset may reflect a fractionation associated with the methylation reaction. Nevertheless, from a practical standpoint, we emphasize that volatility and evaporation are greater concerns than acidic exchange on aromatic compounds, when pursuing phthalic acid methylation or methylation of reagents with similar or lower boiling points. We recommend that analytical conditions be carefully designed and monitored to minimize evaporative losses and further isotopic enrichment, by testing quantitative yields by GC/FID in parallel with isotopic determinations by GC/IRMS. 
In contrast, the non-acidic carbodiimide method performed poorly in phthalic acid methylation experiments in terms of product yield and depletion in both ${ }^{2} \mathrm{H}$ (by up to $15 \%$ ) and ${ }^{13} \mathrm{C}$ (by up to $2 \%$; Figure 3 ). We hypothesize that the low isolated yield is related to the isotopic depletion but the yield could not be improved in our attempts shown here and thus the consequent isotope effects could not be directly tested. Since acidic-exchange is not quantitatively important for these applications, we have no reason to extend testing of the non-acidic method here.

Phthalic acid methylation is commonly used to determine the isotopic composition of methyl groups in methanol used for fatty acid esterification. ${ }^{[19]}$ While the different methods induce isotopic offsets from predicted values in this study (Table 1), the error in the isotopic composition of the methanol becomes trivial for long chain $n$-alkanoic acids due to the small proportion of methyl hydrogens and carbon. For example, a $54 \%$ offset in the methyl $\delta^{2} \mathrm{H}$ value becomes $2 \%$ for the $\delta^{2} \mathrm{H}$ value of $\mathrm{C}_{28}$ FAME and $5 \%$ for $\mathrm{C}_{16}$ FAME and a $7 \%$ offset in the methyl $\delta^{13} \mathrm{C}$ value becomes $0.2 \%$ and $0.3 \%$ for the $\delta^{13} \mathrm{C}$ values of $\mathrm{C}_{28}$ and $\mathrm{C}_{16}$ FAME. These accuracy and precision errors contribute to methyl group uncertainty, and to the propagated uncertainties in the calculation of analyte $\delta^{2} \mathrm{H}$ values. ${ }^{[26]}$ Overall, the magnitude of methyl hydrogen uncertainty on derivatized compounds remains small relative to other sources of uncertainties in the estimation of high molecular weight aliphatic acids e.g., $\mathrm{C}_{28} n$ alkanoic acid, a constituent of plant leaf waxes (Table 1). For short chain lipids such as the $\mathrm{C}_{16}$-alkanoic acid (common in microbial, plant and animal cells) the mean isotope values differ more between methods (up to $5 \%$ ) and uncertainties increase. For practical guidance, we provide detailed protocol and error propagations in the supplementary material.

\section{CONCLUSIONS}


Methyl esterification is a commonly used derivatization procedure for both aromatic and aliphatic compounds with carboxylic acid groups prior to GC analysis. We have studied hydrogen and carbon isotopic fractionations during methylation using two widely used acidic catalysts and one non-acidic method using a carbodiimide. We found practical advantages to the acidic methods over the carbodiimide method, notably higher isolated yields. We determined that the rates of ${ }^{1} \mathrm{H} /{ }^{2} \mathrm{H}$ exchange $\left(4.94 \times 10^{-4} \mathrm{hr}^{-1}\right)$ are too slow to be of quantitative importance and thus find that the acidic conditions do not present problems for most applications, even with aromatic compounds. We thus recommend following one of the acidic protocols.

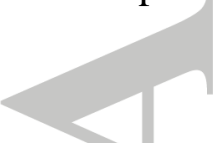

A phthalic acid standard is commonly used to determine the isotopic composition of the methanol used to derivatize both the standard and the aliphatic or aromatic acid analyte for GC/IRMS applications. Using phthalic acid and methanol standards of known values here, we establish that for both carbon and hydrogen isotopic analyses the carbodiimide method produces systematically lower values than the acidic methods. As we have shown that acidicexchange is not quantitatively significant, the greatest issues appear to be low yield (carbodiimide method) and evaporation (under poor laboratory handling scenarios). Although we observe a small systematic ${ }^{13} \mathrm{C}$ - and ${ }^{2} \mathrm{H}$-enrichment in dimethyl phthalate from known reagents using the acidic methods, the propagated errors for long chain $n$-alkanoic acid applications are smaller than the analytical uncertainty of $\delta^{2} \mathrm{H}$ values by GC/IRMS at least for high molecular weight analytes.

\section{ACKNOWLEDGEMENTS}

This work was supported by funding from the US American Chemical Society Award \#PRF53747-ND2 to SF, from the US National Science Foundation Award CHE-1566167 to TW, 
and from the USC Provost fellowship to HJL. Federal support of NMR spectrometers (NSF DBI-0821671, CHE-0840366; NIH S10 RR25432) is gratefully acknowledged. We thank

Elias Karkabi for discussions. This manuscript was improved with the comments from three reviewers.

\section{REFERENCES}

[1] P. E. Sauer, T. I. Eglinton, J. M. Hayes, A. Schimmelmann, A. L. Sessions. Compound-specific D/H ratios of lipid biomarkers from sediments as a proxy for environmental and climatic conditions. Geochim. Cosmochim. Acta 2001, 65, 213.

[2] J. E. Tierney, J. M. Russell, Y. Huang, J. S. S. Damste, E. C. Hopmans, A. S. Cohen. Northern hemisphere controls on tropical southeast African climate during the past 60,000 years. Science 2008, 322, 252.

[3] S. J. Feakins, L. P. Bentley, N. Salinas, A. Shenkin, B. Blonder, G. R. Goldsmith, C. Ponton, L. J. Arvin, M. S. Wu, T. Peters, A. J. West, R. E. Martin, B. J. Enquist, G. P. Asner, Y. Malhi. Plant leaf wax biomarkers capture gradients in hydrogen isotopes of precipitation from the Andes and Amazon. Geochim. Cosmochim. Acta 2016, 182, 155.

[4] L. Gao, E. J. Edwards, Y. Zeng, Y. Huang. Major evolutionary trends in hydrogen isotope fractionation of vascular plant leaf waxes. PLoS One 2014, 9, 1.

[5] K. A. Hobson, L. Atwell, L. I. Wassenaar. Influence of drinking water and diet on the stable-hydrogen isotope ratios of animal tissues. Proc. Natl. Acad. Sci. USA. 1999, 96, 8003.

[6] K. S. Dawson, M. R. Osburn, A. L. Sessions, V. J. Orphan. Metabolic associations with archaea drive shifts in hydrogen isotope fractionation in sulfate-reducing bacterial lipids in cocultures and methane seeps. Geobiology 2015, 13, 462.

[7] A. L. Sessions, T. W. Burgoyne, A. Schimmelmann, J. M. Hayes. Fractionation of 
hydrogen isotopes in lipid biosynthesis. Org. Geochem. 1999, 30, 1193.

[8] M. R. Osburn, A. L. Sessions, C. Pepe-Ranney, J. R. Spear. Hydrogen-isotopic variability in fatty acids from Yellowstone National Park hot spring microbial communities. Geochim. Cosmochim. Acta 2011, 75, 4830.

[9] P. J. Polissar, K. H. Freeman, D. B. Rowley, F. A. McInerney, B. S. Currie. Paleoaltimetry of the Tibetan Plateau from D/H ratios of lipid biomarkers. Earth Planet. Sci. Lett. 2009, 287, 64.

[10] C. Ponton, A. J. West, S. J. Feakins, V. Galy. Leaf wax biomarkers in transit record river catchment composition. Geophys. Res. Lett. 2014, 41, 6420.

[11] A. Schimmelmann, A. L. Sessions, M. Mastalerz. Hydrogen isotopic (D/H) composition of organic matter during diagenesis and thermal maturation. Annu. Rev. Earth Planet. Sci. 2006, 34, 501.

[12] F. Camin, L. Bontempo, L. Ziller, C. Piangiolino, G. Morchio. Stable isotope ratios of carbon and hydrogen to distinguish olive oil from shark squalene-squalane. Rapid Commun. Mass Spectrom. 2010, 24, 1810.

[13] B. J. Tipple, L. A. Chesson, B. R. Erkkila, T. E. Cerling, J. R. Ehleringer. B-HIVE: Beeswax hydrogen isotopes as validation of environment, part II. Compound-specific hydrogen isotope analysis. Food Chem. 2012, 134, 494.

[14] A. L. Sessions, S. P. Sylva, R. E. Summons, J. M. Hayes. Isotopic exchange of carbon-bound hydrogen over geologic timescales. Geochim. Cosmochim. Acta 2004, $68,1545$.

[15] A. L. Sessions. Factors controlling the deuterium contents of sedimentary hydrocarbons. Org. Geochem. 2016, 96, 43.

[16] M. J. DeNiro, S. Epstein. Isotopic composition of cellulose from aquatic organisms. Geochim. Cosmochim. Acta 1981, 45, 1885. 
[17] S. Epstein, C. J. Yapp, J. H. Hall. The determination of the D/H ratio of nonexchangeable hydrogen in cellulose extracted from aquatic and land plants. Earth Planet. Sci. Lett. 1976, 30, 241.

[18] P. A. de Groot. Handbook of Stable Isotope Analytical Techniques. Elsevier, Amsterdam, 2008.

[19] A. L. Sessions. Isotope-ratio detection for gas chromatography. J. Sep. Sci. 2006, 29, 1946.

[20] W. W. Christie. Gas Chromatography and Lipids : A Practical Guide. Oily Press, Ayr, 1989.

[21] H. C. Brown, Y. Okamoto. Electrophilic substituent constants. J. Am. Chem. Soc. 1958, $420,4979$.

[22] G. A. Olah. Mechanism of electrophilic aromatic substitutions. Acc. Chem. Res. 1971, 4, 240.

[23] N. H. Werstiuk, T. Kadai. The high temperature and dilute acid (HTDA) procedure as a general method of replacing aromatic hydrogen by deuterium. II. Can. J. Chem. 1974, $52,2169$.

[24] P. E. Sauer, T. I. Eglinton, J. M. Hayes, A. Schimmelmann, A. L. Sessions. Compound-specific D/H ratios of lipid biomarkers from sediments as a proxy for environmental and climatic conditions. Geochim. Cosmochim. Acta 2001, 65, 213.

[25] A. L. Sessions, L. L. Jahnke, A. Schimmelmann, J. M. Hayes. Hydrogen isotope fractionation in lipids of the methane-oxidizing bacterium Methylococcus capsulatus. Geochim. Cosmochim. Acta 2002, 66, 3955.

[26] P. J. Polissar, W. J. D’Andrea. Uncertainty in paleohydrologic reconstructions from molecular $\delta$ D values. Geochim. Cosmochim. Acta 2014, 129, 146.

[27] F. Lehmann, A. Pettersen, E. A. Currier, V. Sherbukhin, R. Olsson, U. Hacksell, K. 
Luthman. Novel potent and efficacious nonpeptidic urotensin II receptor agonists. $J$. Med. Chem. 2006, 49, 2232.

[28] M. Mikolajczyk, P. Kielbasiński. Recent developments in the carbodiimide chemistry. Tetrahedron 1981, 37, 233.

[29] M. Zhang, P. Vedantham, A. Daniel L. Flynn, P. R. Hanson. High-load, soluble oligomeric carbodiimide: synthesis and application in coupling reactions. J. Org. Chem. 2004, 69, 8340.

[30] J. Sheehan, P. Cruickshank, G. Boshart. Convenient synthesis of water-soluble carbodiimides. J. Org. Chem. 1961, 26, 2525.

[31] B. Neises, W. Steglich. Simple method for the esterification of carboxylic acids. Angew. Chemie Int. Ed. English 1978, 17, 522.

[32] A. L. Sessions, T. W. Burgoyne, J. M. Hayes. Determination of the H3 factor in hydrogen isotope ratio monitoring mass spectrometry. Anal. Chem. 2001, 73, 200.

[33] M. Tsakos, E. S. Schaffert, L. L. Clement, N. L. Villadsen, T. B. Poulsen, M. Sasaki, R. Mynott, C. W. Lehmann, T. Ye, B. A. Littlefield, P. Giannakakou, E. Hamel, K. Watashi, S. Kobayashi, F. Sugawara, K. K. Murthi, L. N. Gentile, J. H. Liu. Ester coupling reactions - an enduring challenge in the chemical synthesis of bioactive natural products. Nat. Prod. Rep. 2015, 32, 605.

[34] A. Höpfner. Vapor pressure isotope effects. Angew. Chemie Int. Ed. English 1969, 8, 689.

[35] J.F. Marlier. Multiple isotope effects on the acyl group transfer reactions of amides and esters. Acc. Chem. Res. 2001, 34, 283.

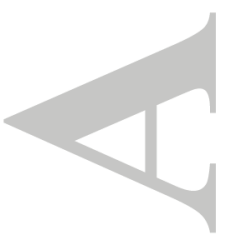




\section{TABLE}

Table 1. Hydrogen and carbon isotope ratios calculated for $\mathrm{C}_{28}$ and $\mathrm{C}_{16}$ FAMEs and propagated uncertainties based on the precision and accuracy of dimethyl phthalate analyses.

\begin{tabular}{|c|c|c|c|c|c|c|}
\hline & \multicolumn{3}{|c|}{$\delta^{2} \mathrm{H}$ values $(\%$ ) } & \multicolumn{3}{|c|}{$\delta^{13} \mathrm{C}$ values $(\%)$} \\
\hline & $\mathrm{HCl}$ & $\mathrm{CH}_{3} \mathrm{COCl}$ & $E D C I / D M A P$ & $\mathrm{HCl}$ & $\mathrm{CH}_{3} \mathrm{COCl}$ & EDCI/DMAP \\
\hline Mean dimethyl phthalate & -107 & -105 & -137 & -31.3 & -31.5 & -32.7 \\
\hline \multicolumn{7}{|l|}{ Uncertainties } \\
\hline i. Precision ${ }^{\dagger}$ & 1 & 2 & 1 & 0.1 & 0.0 & 0.4 \\
\hline ii. Accuracy $^{\circ}$ & 16 & 18 & 14 & 0.2 & 0.4 & 1.6 \\
\hline Estimated methyl group & -115 & -112 & -165 & -47.9 & -48.9 & -54.5 \\
\hline Assigned FA value & & -100 & & & -20 & \\
\hline $\mathrm{C}_{28}$ FAME calculated & -101 & -101 & -103 & -21.0 & -21.0 & -21.2 \\
\hline \multicolumn{7}{|l|}{ Propagated $\sigma *$} \\
\hline i. Precision & 6 & 6 & 6 & 0.2 & 0.2 & 0.2 \\
\hline ii. Accuracy & 6 & 6 & 6 & 0.2 & 0.2 & 0.3 \\
\hline $\mathrm{C}_{16}$ FAME calculated & -101 & -101 & -106 & -21.6 & -21.7 & -22.0 \\
\hline \multicolumn{7}{|l|}{ Propagated $\sigma *$} \\
\hline i. Precision & 6 & 6 & 6 & 0.2 & 0.2 & 0.2 \\
\hline ii. Accuracy & 7 & 7 & 7 & 0.2 & 0.2 & 0.5 \\
\hline
\end{tabular}

${ }^{\dagger}$ Reproducibility of phthalic acid methylation and preparative chemistry.

${ }^{\circ}$ Difference between known and measured dimethyl phthalate value from Figure 3.

* Propagated standard deviation calculated following the approach of Polissar and D'Andrea ${ }^{[26]}$. 


\section{Figure 1.}

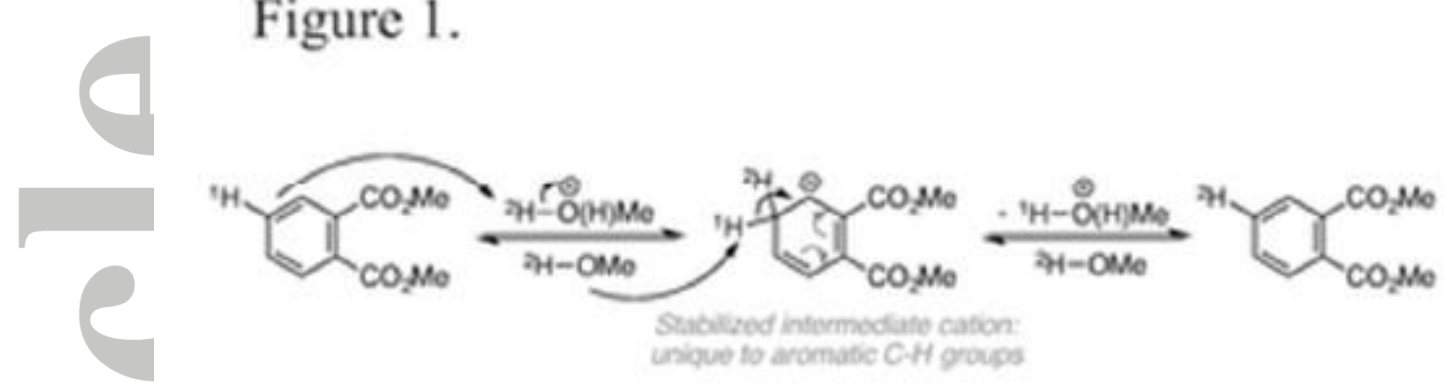

Figure 1. Schematic diagram showing the risk of acid-catalyzed ${ }^{1} \mathrm{H} /{ }^{2} \mathrm{H}$ exchange in dimethyl phthalate.
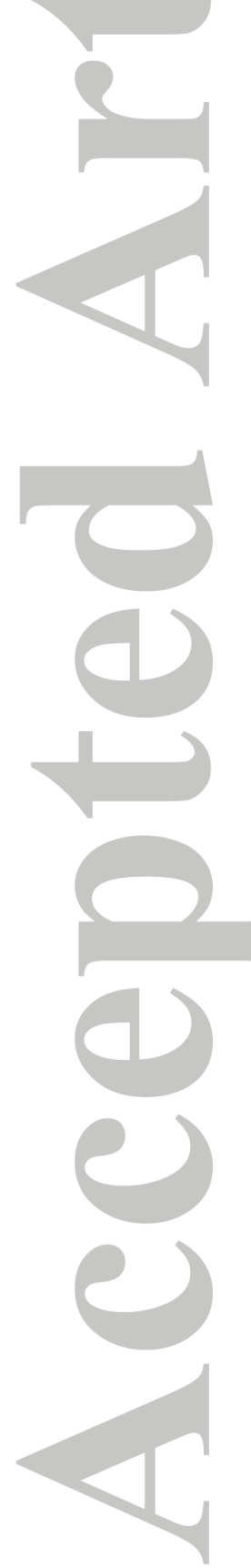


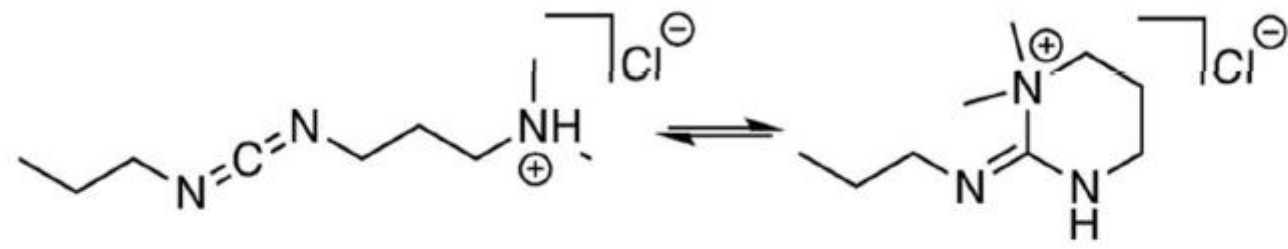

EDCI (N-(3-Dimethylaminopropyl)-N-ethylcarbodiimide hydrochloride)

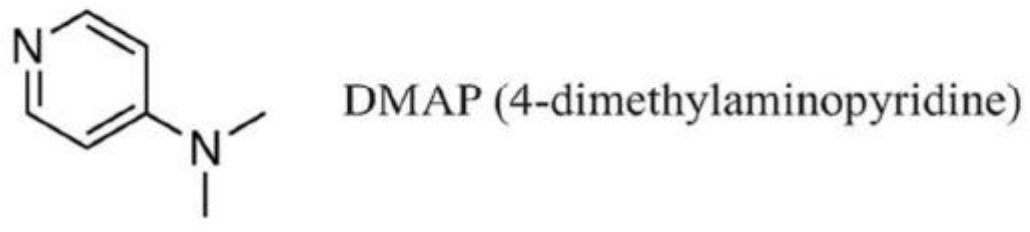

Figure 2. The structures of non-acidic reagents EDCI and DMAP 
Figure 3.
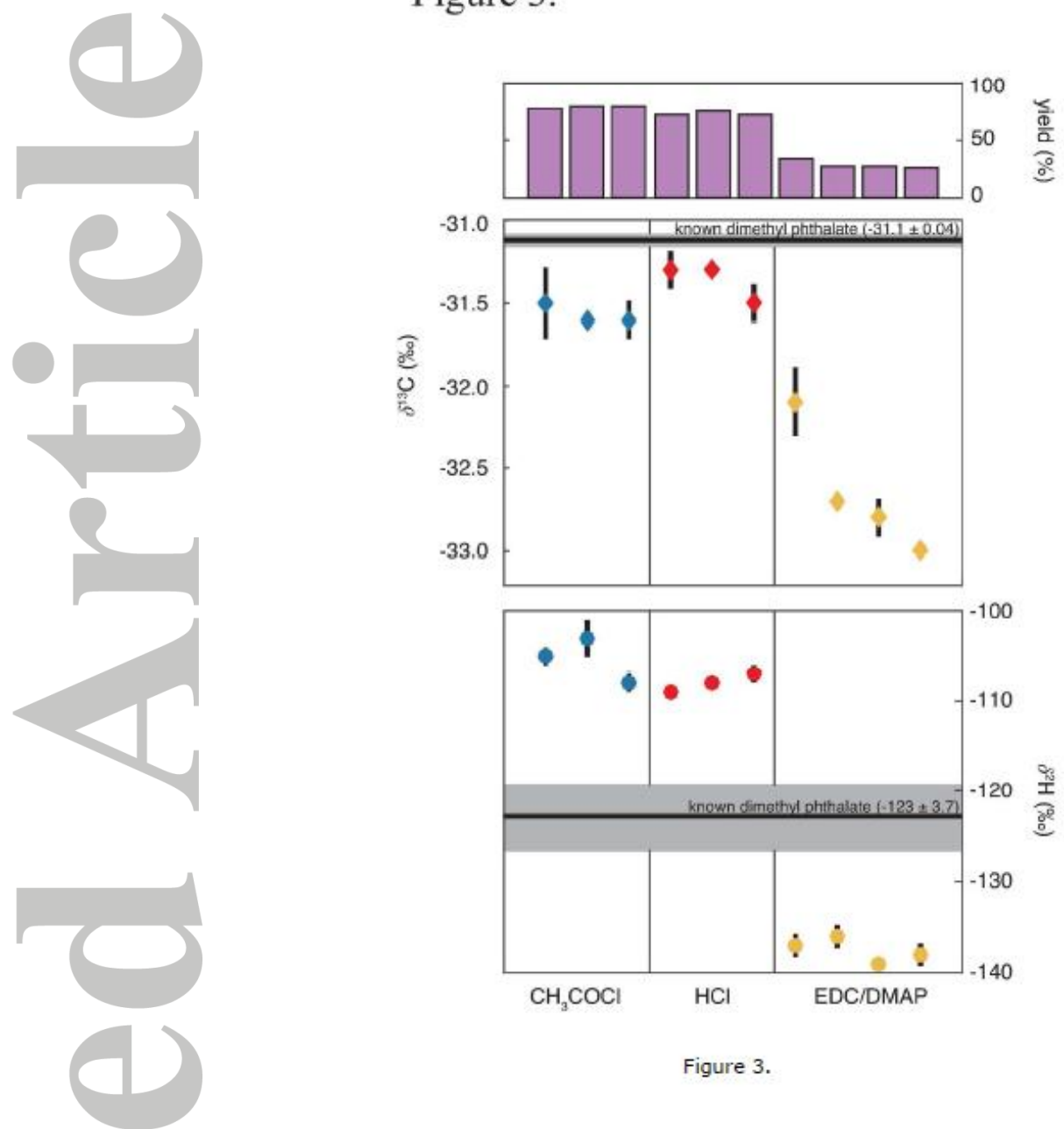

Figure 3. Dimethyl phthalate isolated yield and isotopic results. Carbon and hydrogen isotopic measurements are compared with known dimethyl phthalate values (i.e., target values) calculated by isotopic mass balance. Diamonds represent carbon isotope results whereas circles represent hydrogen isotopes. 


\section{Figure 4.}

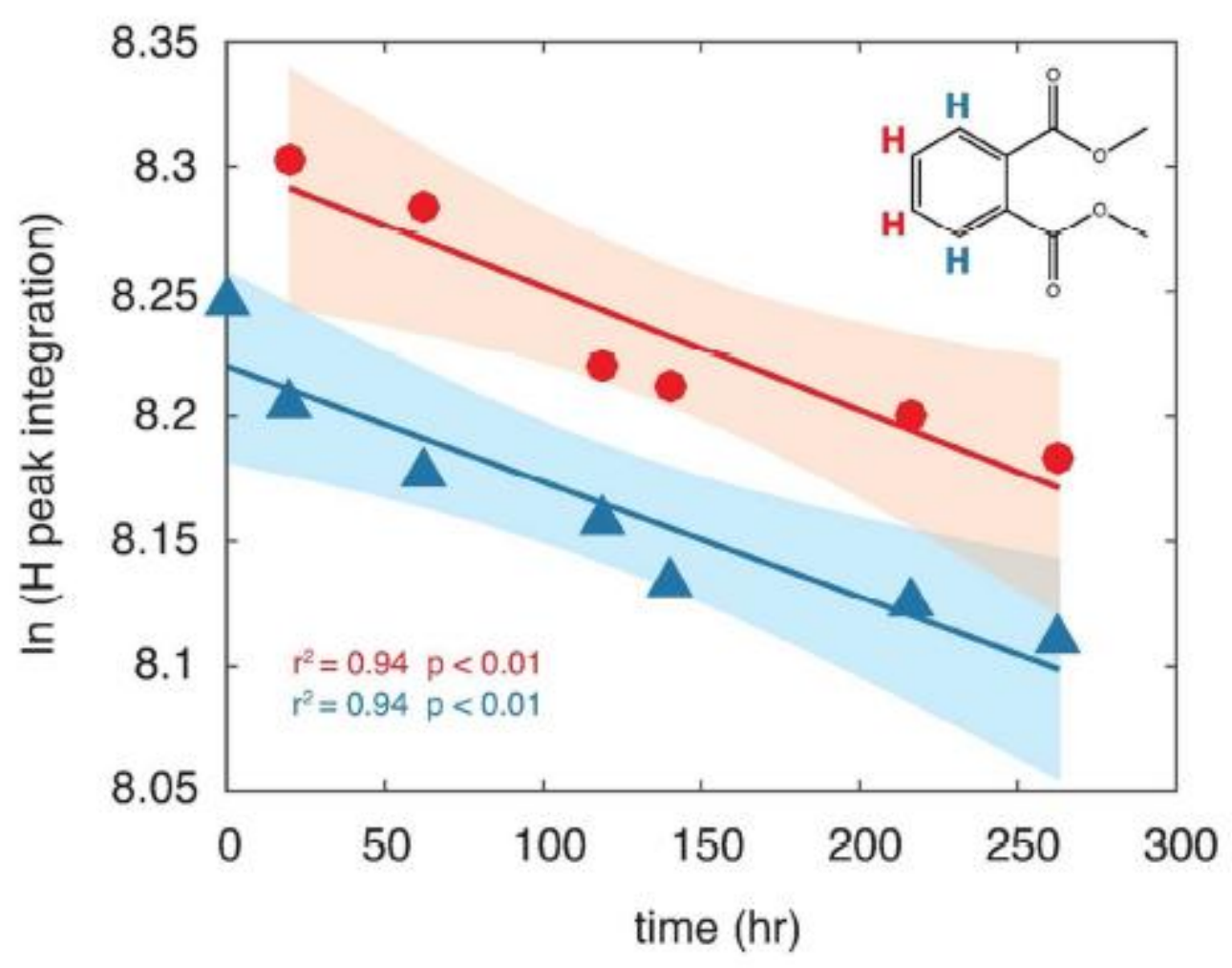

Figure 4. Pseudo-first order rate $\left(k_{\mathrm{nmr}}\right)$ of $\mathrm{HCl}$-mediated ${ }^{1} \mathrm{H} /{ }^{2} \mathrm{H}$ exchange rate in dimethyl phthalate. Hydrogens attached to the beta carbon position (red symbols, red line) and to the alpha carbon (blue triangles, blue line). 
Figure 5.

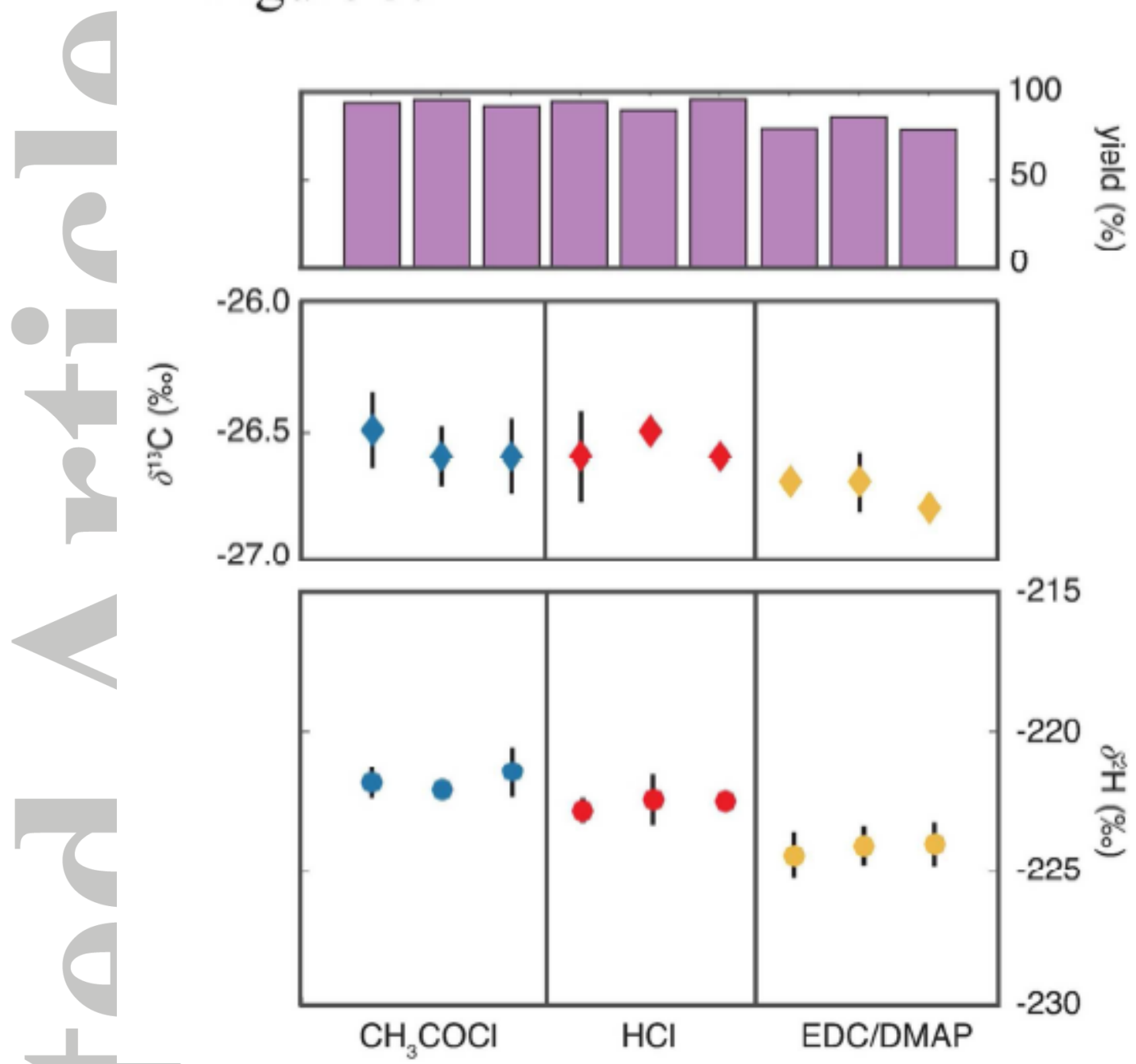

Figure 5. Methyl octacosanoate isolated yield, carbon and hydrogen isotopic composition. Error bars are replicate instrument precision, results are the same within analytical uncertainty. 